\title{
COMUNIDADES ZOOPLANCTONICAS DE LA ALBUFERA DE VALENCIA
}

\author{
R. Oltra y M.R. Miracle \\ Departamento de Ecología. Facultad de Ciencias Biológicas. Universidad de Valencia
}

Palabras Clave: Hyperthrophie coastal lagoon, Zooplanctonic communities, Albufera of Valencia (Spain).

\author{
ABSTRACT \\ ZOOPLANKTONIC COMMUNITIES OF ALBUFERA OF VALENCIA (SPAIN), A COASTAL LAGOON
}

The zooplankton of the Albufera, a large but shallow hypertrophic coastal lagoon, is constituted basicly by the copepod Acantbocyclops vernulis and several rotifer species mainly belonging to the Brachionus genus. Quantitative analysis of samples taken in differenr months and nine localities, which are representative of the distinct zones of the lagoon, show that: (1) the zooplanktonic biomass of this lagoon is very high, being in average arround $2 \mathrm{mg} / \mathrm{l}$, corresponding roughly to 2 individuals $/ \mathrm{ml}$, and (2) the ratio between zooplankton and fitoplankton biomass is very low, arround 1 per one hundred; if rhe numbers of individuals are considered the ratio zooplankton/phytoplankton oscillates arround 2 perone million. Both results are characteristic features of hypertrophic lagoons.

The results of the statistical study of zooplankton counts poinr our the existence of the main associarions or groups of species corresponding to a seasonal succesion: (1) a group constituted by rhe permanent species A. vernalis and Brachionus. angulurir, which are moreover associared with species showing a summer occurrence and a correlation with the salinity of the waters, such as B. plicatilis; (2) a second group, occurring at rhe end of the summer, composed by B. calyciflorus, Anuraeopsis fissa. Asplanchna girodi and Polyarthra dolichoptera. The importance of these groups is very different depending on the distinct localities. Those corresponding to the Northern margin of the lagoon show a predominance of rotifers over the copepods and a much more marked succesion in time rhan the rest. Furthermore the northern localities are notably characterized by the association with B. calyciflorus at the end of the summer, which is almost inexistent in the rest of the ligoon.

\section{INTRODUCCION}

En los últimos años la Albufera de Valencia ha sufrido un proceso de eutrofización de características alarmantes, que ha hecho cambiar la fauna y flora del sistema a un ritmo muy acelerado. Respecto al zooplancton, esto se evidencia al comparar las poblaciones actuales con las registradas en trabajos anteriores (Arévalo 1916, 1918; Pardo 1942; Blanco 1974).

El presente trabajo pretende describir las características actuales del zooplancton de la Albufera. En ésta, debido a su gran extensión y a la heterogeneidad de las aguas que le llegan por distintos

Limnética 1: 51-61 (1984)

(C) Asociación Española de Limnologia, Madrid. Spain puntos, pueden observarse, dentro del sistema general, una serie de subambientes de condiciones distintas, puestas de manifiesto en la diferente composición específica de las comunidades fitoplantónicas Miracle et al (1984).

Por ello, para poder dar una idea lo más completa del zooplancton de la Albufera y su posible diferenciación en las distintas zonas, se han estudiado sus poblaciones en los mismos puntos de muestreo que se eligieron como representativos de los diferentes subambientes en el citado trabajo de Miracle $c t$ al. (1984). En realidad, ambos trabajos fueron simultáneos, formando parte de un estudio más amplio sobre el estado de la Albufera. En el 
presente artículo se ponen además de manifiesto algunas relaciones de plancton animal con otros parámetros físico-químicos y biológicos que describen la importante variación en el tiempo de las aguas de la Albufera, debida a su particular balance hídrico que presenta, en determinados momentos, tasas de renovación del agua muy altas
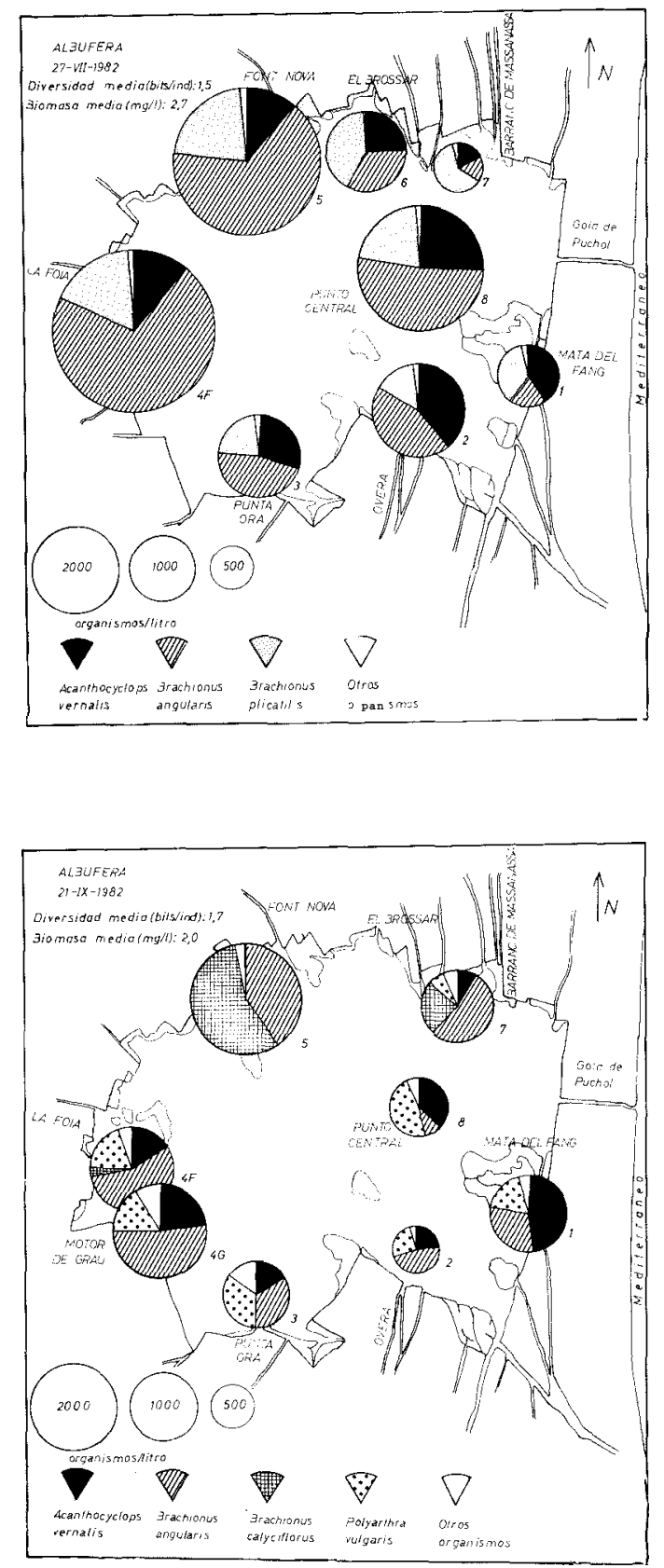

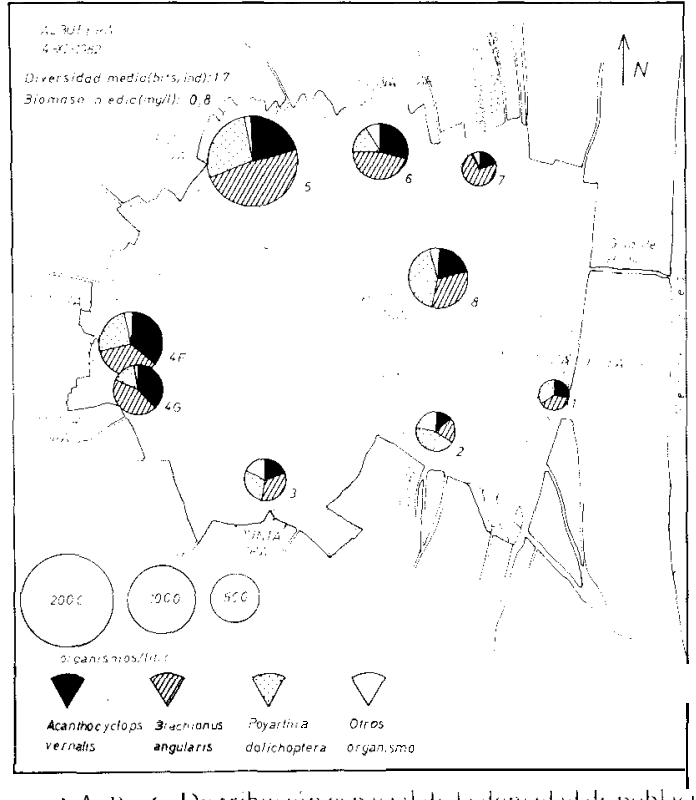

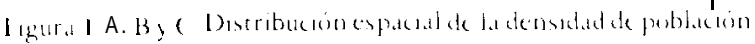
total del zooplanton (el númerodeorganismos/les proporcional al area del círculo) y contribución relativa de las espectes mayoritarías en las fechas indicadas Se señala también la diversidad y biomasa inedia del zooplancton de la laguna para cada dia de muestreo. Spatial distriburion of total population density of zooplankton (the number of orginisms/ I s proportional torhc area of the circle) and relative contribution of the main species in the indicated dates Mean diversity and rnean biomass of the zooplankton of the lagoon are also recorded for each sampling date

\section{MATERIAL Y METODOS.-}

La Albufera de Valencia es una laguna litoral de características oligohalinas, siendo la más extensa y eutrófica de la costa mediterránea española. Se encuentra situada en una zona de huerta a $12 \mathrm{Km}$. al sur de Valencia.

Su superficie oscila alrededor de 2.200 hectáreas y su profundidad media es de unos $75 \mathrm{~cm}$. lo que permite que en algunas zonas afloren densas masas de vegetación lacustre que constituyen verdaderos islotes o "matas". Este carácter somero y la abundancia de vegetación litoral, influyen en el zooplancton favoreciendo la presencia de especies béntico-litorales en la zona pelágica junto a verdaderas especies planctónicas.

Los muestreos se efectuaron en los meses de julio, septiembre y noviembre de 1982 y, en cada uno de ellos, se tomaron muestras en ocho puntos repartidos por las distintas zonas de la laguna (Fig. 1). Las muestras fueron recogidas, en todos los casos, entre las 9 y 14 horas. 
Junto con las muestras de zooplancton, se recogieron otras para la determinación de diversos parámetros físico-químicos, según los métodos descritos en Golterman et al. 1978 y Wetzel y Likens, 1979. Al mismo tiempo se efectuaron mediciones in situ de varios parámetros físico-químicos empleando para ello un salinómetro YSI mod. 33, oxímetro YSI mod. 518 -para medidas de oxígeno, que se confirmaron con análisis por el método Winkler- y un Radiometer 80 para $\mathrm{pH}$ y potencial redox cuyos valores fueron estandarizados a la temperatura de 25 grados centigrados La transparencia del agua se determinó con ayuda del disco de Secchi.

Las muestras destinadas al recuento e identificación del zooplancton, se tomaron a 0,5 metros de profundidad con una botella hidrográfica "Ruttner" de 2'6 litros de capacidad; se filtraron a través de una malla de Nytal de 45 micras de poro, depositándose los filtros en tubos que contenían una solución de formol al $5 \%$, con el fin de fijar los organismos recogidos. Posteriormente se procedió a la clasificación y recuento de los componentes zooplanctónicos sedimentados en cubetas cilíndricas, mediante un microscopio invertido a 100 aumentos (Miracle 1974).

El cálculo de la biomasa del zooplancton se realizó multiplicando el número de individuos de cada grupo por su volumen medio, el cual se calculó, para cada especie y día de muestreo, con las dimensiones medias de los individuos de las diversas poblaciones, asimilando cada una de las especies a una figura geométrica (Edmondson y Winberg 1971; Ruttner-

Tabla I. Densidad de población (enindividuos/l) de las especies del zooplancton de la Albufera en los distintos días de muestreo ylocalidades (cuya situación se indica en la Fig l)

Population density (in individuals/l) of the man zooplanktonic species ot the Albufera lagoon 1 n rhe different sampling dates and localitees (rheir siturtion is indicates in Fig 1 )

\begin{tabular}{|c|c|c|c|c|c|c|c|c|c|c|c|c|c|c|c|c|c|c|c|c|c|c|c|c|c|}
\hline \multirow{2}{*}{$\begin{array}{l}\text { Fecha } \\
\text { Incalidar }\end{array}$} & \multicolumn{8}{|c|}{27 Julio 1982} & \multicolumn{8}{|c|}{ 21. Septientre 10:82 } & \multicolumn{9}{|c|}{4 Novientre 1982} \\
\hline & 1 & 2 & 3 & $4 \pi^{5}$ & 5 & 6 & 7 & 8 & 1 & 2 & 34 & 45 & $4 G$ & 5 & 7 & 8 & 1 & 2 & 3 & $4 \mathrm{~F}$ & 46 & 5 & 6 & 7 & $\overline{8}$ \\
\hline Acanthocyclops vernal is & 387 & 915 & 519 & 7806 & 6694 & 4331 & 1291 & 1065 & 771 & 142 & 3393 & 3115 & 521 & $29 \mathrm{I}$ & 1203 & 348 & 65 & 47 & 75 & 356 & 211 & 304 & 211 & 58 & 176 \\
\hline Mina brachiata & 0 & 0 & 0 & 0 & 0 & 0 & 0 & 0 & 5 & 26 & 69 & 5 & 2 & 84 & 3 & 2 & 0 & 0 & 0 & 0 & 0 & 0 & 0 & 0 & 0 \\
\hline Brachionus angularis & 188 & 1035 & 8404 & 499138 & 30005 & 577 & 952 & 2180 & 465 & 299 & 41010 & 108312 & 23012 & 2497 & 7451 & 02 & 89 & 83 & 128 & 321 & 261 & $905:$ & 340 & 138 & 263 \\
\hline Brachionus plicatil is & 353 & 365 & 4001 & 122813 & 13147 & $738 \quad 4$ & 409 & 908 & 20 & 10 & 19 & 22 & 18 & 92 & 252 & 20 & 0 & 1 & 0 & 0 & 0 & 0 & 0 & 0 & 0 \\
\hline Brachionus calyciflorus & 0 & 0 & 0 & 2 & 2 & + & 0 & 0 & 0 & 0 & + & 65 & 2518 & $822 \quad 34$ & 342 & 0 & 0 & 1 & 1 & 1 & $0 \quad 0$ & $0 \quad 0$ & 3 & 1 & 0 \\
\hline Brachiomus bidentata & 3 & 24 & 3 & 14 & 3 & 19 & 98 & 1 & 0 & 0 & 0 & 0 & 0 & 0 & 0 & 0 & 0 & 0 & 0 & 0 & 0 & 1 & 1 & 0 & 0 \\
\hline Brachionus unceolaris & 0 & 0 & 0 & 0 & 0 & 0 & 0 & 0 & 0 & 0 & 0 & 0 & 0 & + & 0 & 0 & 2 & + & 0 & 0 & 0 & 2 & 0 & 5 & 0 \\
\hline Brachionus quadridentatus & 0 & 0 & 0 & 0 & 0 & 3 & + & 8 & 5 & 6 & + & 3 & 0 & 3 & 10 & 0 & 1 & 0 & 0 & 3 & 0 & 1 & 5 & 2 & 0 \\
\hline Polyarthra vulgaris & 3 & 14 & 28 & 37 & 11 & 3 & 2 & 18 & 283 & 158 & 4183 & 3723 & 373 & 159 & 9245 & 154 & 0 & 0 & 0 & 0 & 0 & 0 & 0 & 0 & 0 \\
\hline Polyarthra dolichoptera & 0 & 0 & 0 & 0 & 0 & 0 & 0 & 0 & 0 & 0 & 0 & 0 & 0 & 0 & 0 & 0 & 73 & 170 & 112 & 232 & 95 & 527 & 115 & 103 & 339 \\
\hline Asplanchria girodi & 2 & 8 & 4 & 0 & 0 & 0 & 5 & 2 & 6 & 71 & 65 & 61 & 32 & 261 & 12 & 4 & 0 & 0 & 0 & 2 & 2 & 3 & 0 & 0 & 1 \\
\hline Synchaeta trenula kitina & 0 & 0 & 0 & 0 & 0 & 0 & 0 & 0 & 0 & 0 & 0 & 0 & 0 & 0 & 0 & 0 & 5 & 73 & 73 & 111 & 173 & 335 & 54 & 5 & 34 \\
\hline Anuraeopsis fissa & 0 & 0 & 0 & 0 & 0 & 0 & 0 & 0 & 0 & 00 & 09 & 2 & 51 & 12 & 0 & 0 & 0 & 0 & 0 & + & 0 & 0 & 0 & 0 & 0 \\
\hline Lecane closterocerca & 1 & 0 & 0 & 0 & 0 & 0 & 0 & 0 & 5 & 0 & 6 & 0 & 0 & 0 & 0 & 0 & 1 & 0 & 0 & 0 & 2 & 0 & 0 & 0 & 0 \\
\hline Lecane bulla & 1 & 0 & 2 & 0 & 0 & + & + & 0 & 3 & 0 & 0 & 0 & 0 & 0 & 0 & 0 & 0 & 0 & 0 & 0 & 0 & 0 & 0 & 1 & 0 \\
\hline Lecane luna & 0 & 1 & 0 & 5 & 0 & + & 0 & 0 & 3 & + & 0 & 0 & 0 & 0 & 3 & 0 & 0 & 0 & 0 & 0 & 0 & 0 & 0 & 0 & 0 \\
\hline Bdel loidea & 0 & 0 & 0 & 0 & 0 & 0 & 7 & 0 & 28 & 3 & 0 & 61 & 33 & 71 & 51 & 6 & 0 & 1 & 0 & 0 & 0 & 2 & 0 & 0 & 0 \\
\hline Ciliata & 0 & 0 & 0 & 0 & 0 & 0 & 2 & 0 & 2 & 60 & 0 & 3 & 63 & 31 & 5 & 9 & 0 & 101 & 25 & 0 & 0 & 5 & 0 & 5 & 0 \\
\hline
\end{tabular}

Resto de las especies, con indicación de: localidad/dia-mes/(densidad en individuos/1) para cada una de sus ocurrencias. (+) indica densidades inferiores a 1.- Rest of the species idicating: station/day-month/(density in ind./1) for each one of their ocurrenres. ( + ) indicates densities lower than 1 .

Keratel la quadrata: $1 / 7-V I I /(1) ; 2 / 21-1 \times /(5)$. Keratella cochlearis: $1 / 4-X I /(1) ; 2 / 4 \times 1 /(1) ; 4 G / 4 \times I /(2)$. Trichocerca pusilla: $2 / 4-X I /(1) ; 3 / 4-X I /(+) ; 5 / 4-X I /(1)$. Lepadella rhomboides: $1 / 21-[X /(14) ; 2 / 21-I X /(1)$. Lecane hamata: $1 / 2]-[X /(2) ; 4 G / 4 X I /(1)$. L. stenroos $1: 1 / 4 \times 1 /(+)$. L. lunaris: 5/4-XI/(2). Ptygura sp.: $4 F / 21-[X /(+)$. 


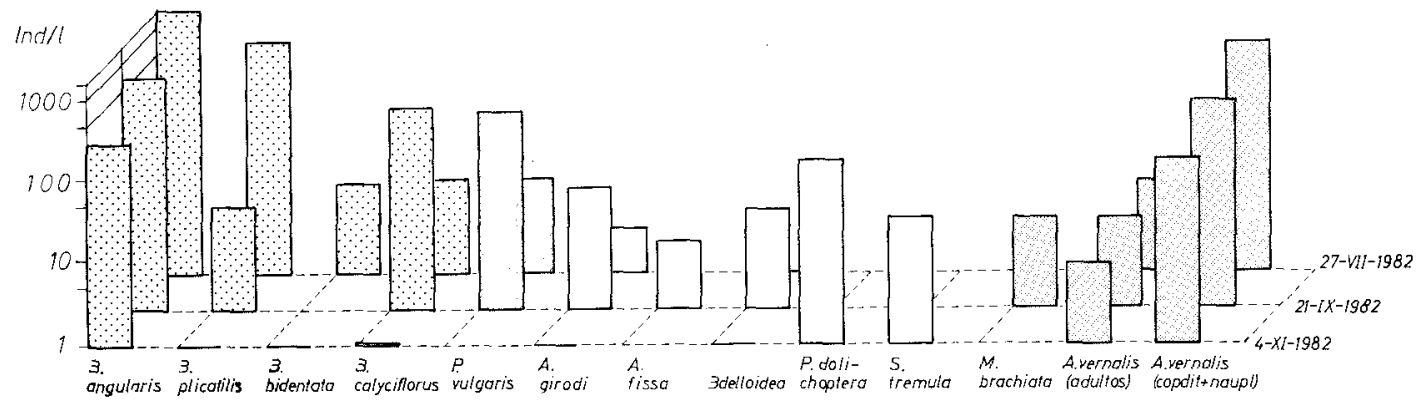

Figura 2- Variación temporal de las principales especies del zooplancton. Los histogramas representan el número medio de individuos por litro (media de las densidades de todas las localidades muestreadas).

Temporal variation of the main species of rhe zooplankton Histograms represenr rhe mean number of individuals per 1 (mean of the desiries for all sampling stations.

Kolisko 1977)

\section{RESULTADOS Y DISCUSION}

En la Tabla 1 se registran las densidades de población que presentan las ocho localidades muestreadas. A la vista de los resultados se desprende que, en el período de estudio, el zooplancton de la Albufera está constituído principalmente por el copépodo Acanthocyclops vernalis -estadios juveniles sobre todo- y por rotíferos pertenencientes a los géneros Brachionus, Polyarthra y Synchaetu. Los cladóceros están muy pobremente representados, habiéndose encontrado únicamente algunos ejemplares del género Mozna. Por último, son abundantes también los ciliados, como cabía esperar en una laguna eutrófica, aunque probablemente su importancia real ha sido subestimada dado que las técnicas de fijación y muestreo utilizadas en este trabajo, son apropiadas fundamentalmente para los metazoos, los cuales constituyen el objeto principal en la mayoría de trabajos sobre el zooplancton.

La densidad de población, expresada como el número medio de organismos por litro, es mucho mayor en verano que en los meses otoñales, siendo de 3.091 org./1. en julio, aproximadamente el doble que la de septiembre -1664 org/l- que a su vez, duplica la de noviembre $-690 \mathrm{org} / 1$.

El copépodo $A$. vernalis, siguiendo la tónica general, es más abundante en el mes de julio (Figuras 1 y 2), en el que la población está constituída mayoritariamente por nauplios y copepoditos, para descender notablemente a medida que avanza el otoño, si bien el número de adultos permanece más constante. El porcentaje de hembras ovígeras es también mayor en julio y disminuye hasta hacerse nulo en noviembre; la producción de huevos, estimada a partir del número de hembras ovígeras, lógicamente presenta una disminución paralela a la de éstas. En el mes de septiembre se dan los mayores porcentajes de adultos -casi todos hembras- portadores de epibiontes del género Epistilis. El rotífero Brachionus angularis es también una especies permanente, que alcanza elevadas cifras en julio, disminuyendo en otoño; en la Albufera se aproxima a densidades de 5.000 individuos/litro y es la especie dominante en los tres meses estudiados. Ademas B. plicatilis y B. bidentata son también más abundantes en julio, desapareciendo prácticamente del plancton en otoño.

En septiembre el zooplancton cambia considerablemente. Así el cladócero Moina brachiata sólo aparece en este mes, aunque en bajas densidades excepto en las estaciones del Norte 5 y 7 , en las que es más abundante; también se ha encontrado algún ejemplar de la forma $M$. micrura si bien su proporción, sin duda baja, no se ha podido determinar. Los rotíferos más abundantes en este mes, además de B. angularis son B. calyciflorus, B. quadridentatus, Polyartbra tolgaris, Asplachna girodi y Anuraeopsis firsa.

Por último, las especies que predominan en noviembre -exceptuando las permanentes comentadas anteriormente- son $\mathrm{P}$. dolichoptera $y$ Synchaeta tremula kitina. junto a las que aparecen en muy baja proporción Keratella cochlearis, $K$. 
quadrata. Trichocerca pusilla y otras especies procedentes de los meses anteriores que se mantienen con poblaciones muy reducidas.

Los resultados del análisis estadístico de las muestras de las ocho localidades en las tres épocas distintas, ponen de manifiesto la existencia de tres asociaciones o grupos de especies que corresponden a la variación en el tiempo descrita anteriomente, no diferenciando prácticamente las ocho localidades. Son resultados muy distintos de los obtenidos con las asociaciones de especies del fitoplancton (Miracle et al. (1984) que caracterizan las localidades.

En la figura 3 pueden observarse estos tres grupos, que se deducen simplemente de un análisis de las correlaciones entre las especies y que son los siguientes:
1) Grupo de las especies estivales-permanentes A. vernalis, B. angularis, B. plicatilis y B. bidentata.

2) Grupo de las especies de finales de verano: $M$. brachiata. B. calyciflorus, A. fissa, A. girodiy $P$. i.ulgaris.

3 ) Grupo de las especies propiamente otoñales( S. tremula y $P$. dolichoptera

Esta variaciones en el tiempo son debidas además de a la disminución de la temperatura, a los cambios causados por el particular régimen hídrico de la Albufera descrito en este mismo volúmen por Serra et al. (1984) y Miracle et al. (1984).Después de mantener las compuertas cerradas durante todo el verano, restringuiendo el flujo de agua, se abren en septiembre para cosechar el arroz, con lo que aumenta el caudal de salida, re-

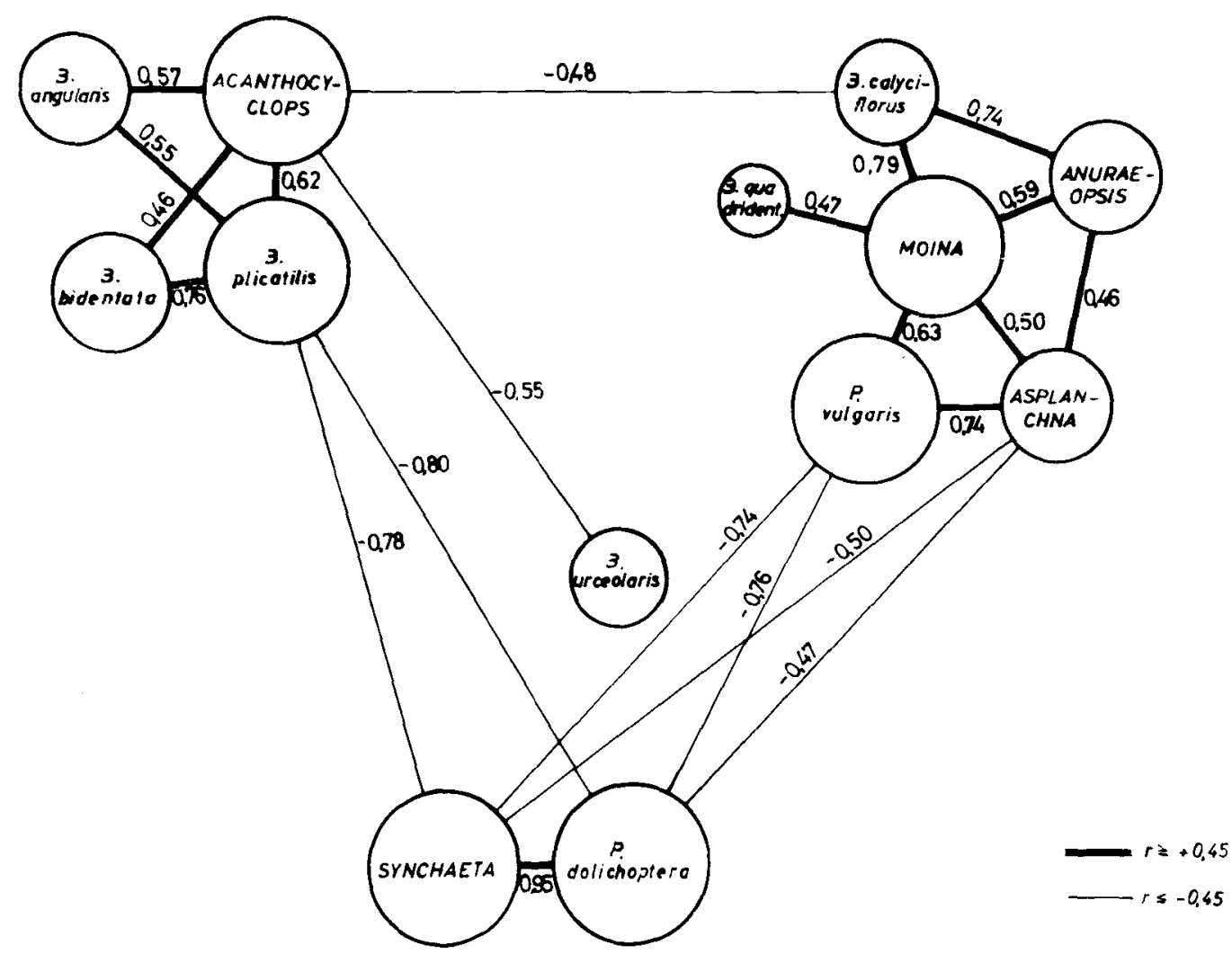

Figura i Agrupación de las especies del zooplanctun según sus coetictences de correlación Loscirculos correspondientes a cada especie tıenen un tamaño proporcional a su coeficiente de correlación múlriple y se enlazan por líneas cuya longitud es indicación del grado de correlación enrre cada par de especiel Sólo se han representado los coeficientes superiores al valor absoluto de 0,44 Clusters of zooplankon spectes according to thrir correlation coefficients the circles corresponding to each species have rheir size proportional to their multiple correlation cotficients and are joined by lines whone lenth ss a rough indication of the degree of correlation berween each parr of species Only correlations exceeding the absoluce value of 0.44 have been represented 


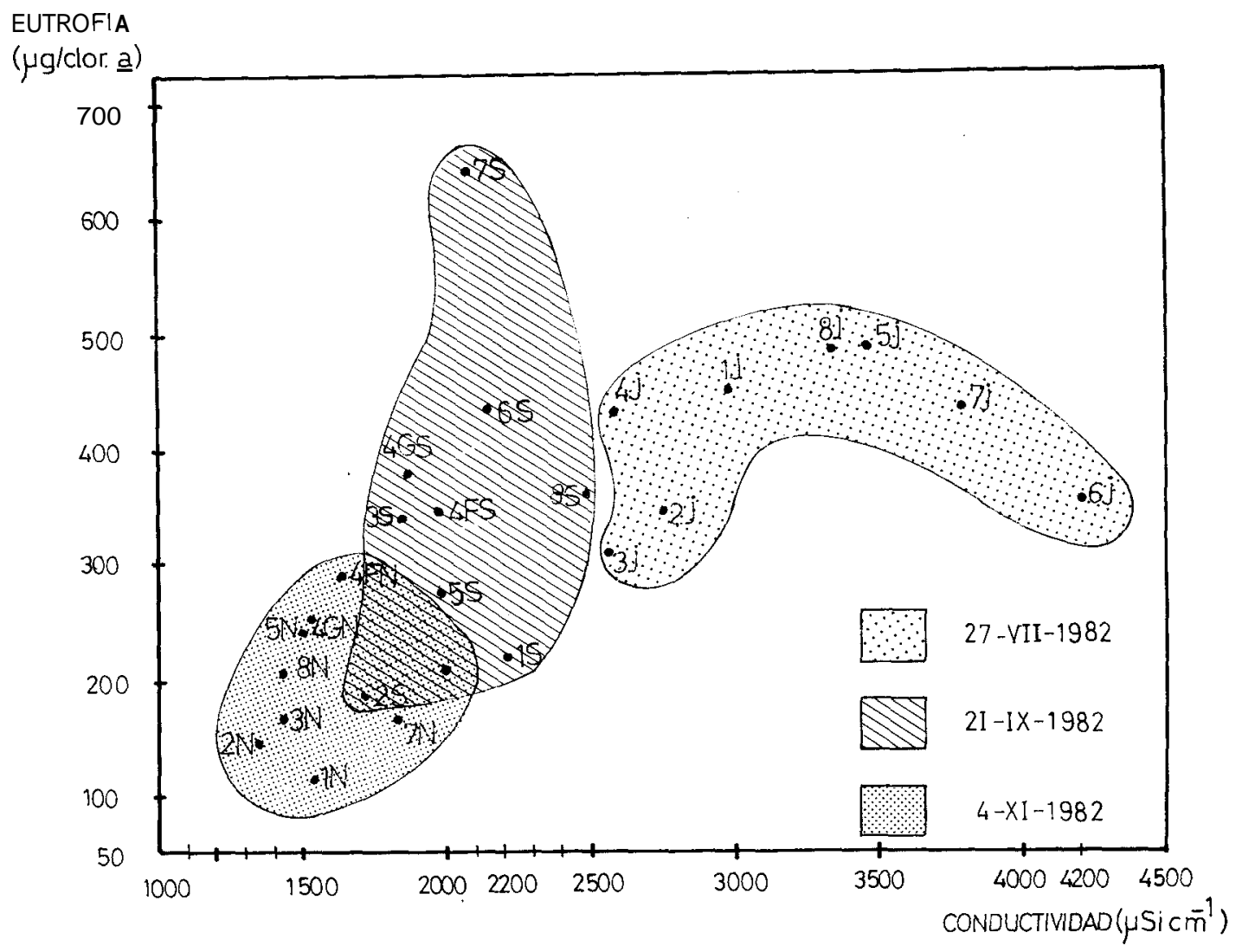

Figura 1 - Representación de las distintas muestras según los dos ejes de mayor variabilidad: contenido de clorofila a y salinidad. Se han agrupado las muestras correspondientes a un mismo día de muestreo.

Representation of the different samples according to the two main variation axes. chlorophyll a contents and salinity. Samples corresponding to the same sampling date have been grouped together

flejandose en una menor conductividad y menor densidad zooplanctónica. El muestreo de noviembre fue realizado después de una importante inundación ocurrida el 20 de octubre de 1.982, que renovó el agua de la laguna, especialmente en las zonas Sur y Este (puntos 1, 2, 3 y 7) debido a la notable afluencia de agua por las acequías allí situadas -principalmente la de Overa: y su salida por la gola de Puchol, con la consiguiente dsiminución de la conductividad y densidad planctónica. En la figura 4 se representan las muestras en los dos ejes de variación más importante, clorofila y conductividad, observándose claramente los fenómenos descritos. Todo ello se refleja en las relaciones entre el zooplancton y diversos parámetros físico-químicos que aparecen ordenadas en la Tabla 2 según el coeficiente de correlación. Se observa como se separan dos grupos de especies: unas correlacionadas fuertemente con la temperatura y conductividad y otras con correlaciones negativas o poco importantes con esos mismos parámetros, lo que evidencia la segregación en el tiempo descrita. Es interesante destacar como se separan las diversas especies del género Brachionus y como se confirma la marcada dependencia descrita en la literatura que presenta B. plicatilis respecto a la conductividad (Ruttner Kolisko 1974; Koste 1978).

La composición zooplanrónica en los ocho puntos de muestreo, se representa en la Fig. 1 Tabla 1. La 
Tabla II - Coeficiente de correlación de las principales especies zooplanctónicas, la contribución relativa de los distintos grupos de organismos y la biomasa y densidad total del zooplancton, con parámetros limnológicos seleccionados entre los que mostraban mayores coeficientes. Se han subrayado las correlaciones superiores a 0,45

Correlation coefficients of the main zooplanctonic species, the relative contribution of crustacea and rotifera and zooplankton biomass and density, with selected limnological parameters among those which gave higher coefficients Correlations greater than 0,45 have been underlined

\begin{tabular}{|c|c|c|c|c|c|c|c|c|}
\hline & $\frac{0}{0}$ & $\ddot{F}$ & I & $\bar{\omega}$ & 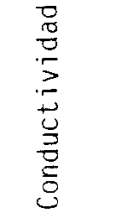 & 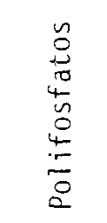 & $m^{m}$ & 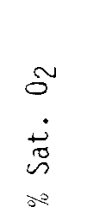 \\
\hline Acanthocyclops vernalis & $\underline{0,56}$ & $\underline{0,62}$ & $\underline{0,48}$ & $-\underline{-0,56}$ & $\underline{0,55}$ & 0,44 & $-0,46$ & $-0,46$ \\
\hline Moina brachi ata & 0,27 & $-0,04$ & 0,42 & 0,15 & $-0,13$ & 0,17 & 0,07 & $\underline{0,53}$ \\
\hline Brachionus angularis & $\underline{0,55}$ & $\underline{0,54}$ & $\underline{0,52}$ & $-0,57$ & 0,39 & 0,42 & $-0,28$ & 0,03 \\
\hline Brachionus plicatilis & $\underline{0,76}$ & $\underline{0,98}$ & 0,66 & $-0,90$ & 0,90 & $\underline{0,54}$ & $-0,75$ & $-0,22$ \\
\hline Brachionus bidentata & 0,53 & 0,74 & 0,30 & $-0,78$ & $\underline{0,76}$ & $\underline{0,55}$ & $-0,09$ & $-0,44$ \\
\hline Brachionus urceolaris & $-0,43$ & $-0,38$ & $-0,42$ & 0,34 & $-0,33$ & $-0,28$ & $\underline{0,64}$ & $-0,11$ \\
\hline Brachionus calycif lorus & 0,17 & $-0,10$ & 0,27 & 0,13 & $-0,75$ & 0,03 & 0,09 & 0,35 \\
\hline Polyarthra vulgaris & 0,45 & 0,43 & $\underline{0,66}$ & $-0,28$ & 0,24 & 0,42 & $-0,18$ & 0,35 \\
\hline Polyarthra dolichoptera & $-\underline{0,65}$ & $-0,82$ & $-0,69$ & 0,67 & $-0,72$ & $-0,55$ & $-0,08$ & 0,04 \\
\hline Synchaeta tremula & $-0,66$ & $-0,81$ & $-\underline{0,68}$ & $\underline{0,66}$ & $-0,70$ & $-\underline{0,53}$ & $-0,08$ & $-0,05$ \\
\hline Aspl anchna girodi & 0,28 & 0,20 & 0,42 & $-0,04$ & 0,06 & 0,13 & $-0,03$ & 0,40 \\
\hline Anuraeopsis fissa & 0,04 & $-0,05$ & 0,23 & 0,08 & $-0,11$ & 0 & 0,11 & 0,25 \\
\hline$\%$ de Rotíferos & 0,32 & 0,70 & 0,50 & $-0,64$ & 0,77 & 0,28 & $-0,01$ & $-0,27$ \\
\hline "A de Crustáceos & $-0,27$ & $-0,72$ & $-0,43$ & 0,65 & $-0,73$ & $-\underline{E}$ & $-0,02$ & 0,33 \\
\hline Biomasa zooplancton & 0,44 & 0,68 & 0,66 & $-0,56$ & 0,57 & 0,56 & $-0,30$ & 0,07 \\
\hline № individuos zooplancton & 0,39 & $\underline{0, \sim}$ ! & 2.05 & $-\underline{0,62}$ & 0,53 & 0,49 & $-0,33$ & 0,07 \\
\hline
\end{tabular}

Tabla 3 resume las principales características de dichas localidades, siendo muy fluctuantes las relacionadas con la producción primaria. Las estaciones del Norte $(5,6$ y 7$)$ tienen carácter más eutrófico. Si bien las variaciones en las comunidades zooplantónicas son mayores en el tiempo que en las diversas zonas de la Albufera, existen algunas diferencias en las localidades estudiadas bastante acordes con las presentadas por el fitoplancton (Miracle et al. 1984).

La estación 7 se presenta claramente diferenciada de las demás mostrando las siguientes particularida- des: 1) una extremada preponderancia de los rotíferos sobre los copépodos; 2 ) La presencia de M. brachiata que llega a ser un $3 \%$ en Septiembre, cuando en otros puntos es muy inferior al $1 \%$ o está ausente; 3 ) presentar importantes poblaciones de los rotíferos B. plicatilis y B. calyciflorus, mientras que en otras zonas B. angularis es siempre el dominante y finalmente una escasa abundancia de las poblaciones del género Polyartbra.

Las estaciones 5 y 6 , igualmente de la zona Norte, presentan un zooplanton bien caracterízado y con 
Tabla III - Características principales de las nueve localidades muestreadas Los datos corresponden a los valores de superficie en las fechas indicadas.

Selected fearures of rhe nime sampling stations. Data are the surface values in rhe indicared dates

\begin{tabular}{|c|c|c|c|c|c|c|c|c|c|c|}
\hline & \multirow[b]{2}{*}{ Fecha } & \multicolumn{9}{|c|}{ Localidad } \\
\hline & & 1 & 2 & 3 & $4 G$ & $4 \mathrm{~F}$ & 5 & 6 & 7 & 8 \\
\hline \multirow{3}{*}{$\begin{array}{c}\text { Temperatura } \\
{ }^{\circ} \mathrm{C}\end{array}$} & $27-7-82$ & 30 & 30 & 30,5 & - & 30 & 30 & 27,8 & 28 & 30 \\
\hline & $21-9-82$ & 23 & 21,5 & 21 & 22 & 22 & 22 & 22,5 & 23 & 23 \\
\hline & $4-11-82$ & 18 & 16,5 & 16,2 & 17 & 16,5 & 17,5 & 18 & 18 & 18,5 \\
\hline \multirow[t]{2}{*}{$\underset{\%}{\text { Sal inidad }}$} & $\begin{array}{l}27-7-82 \\
21-9-82\end{array}$ & $\begin{array}{l}1,9 \\
1,5\end{array}$ & $\begin{array}{l}1,9 \\
1,0\end{array}$ & $\begin{array}{l}1,8 \\
1,1\end{array}$ & $\overline{1,2}$ & $\begin{array}{l}2,0 \\
1,5\end{array}$ & $\begin{array}{l}2,2 \\
1,5\end{array}$ & $\begin{array}{l}2,8 \\
1,5\end{array}$ & $\begin{array}{l}2,5 \\
1,8\end{array}$ & $\begin{array}{l}2,0 \\
2,0\end{array}$ \\
\hline & $4-11-82$ & 1,0 & 0,5 & 0,8 & 1,0 & 1,0 & 1,0 & 1,4 & 1,2 & 1,0 \\
\hline \multirow{3}{*}{$\mathrm{pH}$} & $27-7-82$ & 9,1 & 9,0 & 8,9 & - & 8,8 & 9,5 & 8,6 & 8,4 & 9,3 \\
\hline & $21-9-82$ & 8,9 & 8,4 & 8,8 & 9,2 & 9,1 & 9,0 & 9,7 & 9,3 & 9,2 \\
\hline & $4-11-82$ & 8,6 & 8,2 & 8,2 & 8,0 & 8,1 & 8,2 & 8,4 & 7,7 & 8,9 \\
\hline \multirow{3}{*}{ Eh } & $27-7-82$ & 254,0 & 261,0 & 263,0 & - & 255,0 & 241,0 & 271,0 & 270,0 & 298,0 \\
\hline & $21-9-82$ & 341,0 & 322,0 & 315,0 & 302,0 & 321,0 & 322,0 & 323,0 & 331,0 & 321,0 \\
\hline & $4-11-82$ & 371,0 & 404,0 & 394,0 & 376,0 & 385,0 & 369,0 & 370,0 & 377,0 & 370,0 \\
\hline \multirow{3}{*}{$\begin{array}{c}\text { Oxigeno } \\
\mathrm{mg} / 1\end{array}$} & $27-7-82$ & 6,1 & 9,1 & 13,3 & - & 7,5 & 5,2 & - & 6,1 & 5,8 \\
\hline & $21-9-82$ & 9,7 & 9,8 & 9,8 & $9, \hat{3}$ & 10,6 & 12,2 & - & 15,5 & 11,2 \\
\hline & $4-11-82$ & 12,2 & 10,0 & 9,2 & 9,8 & 12,8 & 11,9 & - & 5,0 & 11,5 \\
\hline \multirow{3}{*}{$\begin{array}{c}\text { Secchi } \\
\mathrm{m}\end{array}$} & $27-7-82$ & 0,25 & 0,28 & 0,28 & - & 0,22 & 0,22 & 0,25 & 0,2 & 0,25 \\
\hline & $21-9-82$ & 0,32 & 0,32 & 0,28 & 0,26 & 0,32 & 0,32 & 0,35 & 0,17 & 0,37 \\
\hline & $4-11-82$ & 0,22 & 0,25 & 0,19 & 0,20 & 0,21 & 0,21 & 0,21 & 0,21 & 0,20 \\
\hline \multirow{3}{*}{$\begin{array}{l}\% \text { Cianobacter. } \\
\text { en biornasa }\end{array}$} & $27-7-82$ & 97,2 & 97,5 & 98,5 & - & 96,9 & 94,8 & 90,5 & 61,8 & 92,8 \\
\hline & $21-9-82$ & 85,7 & 91,9 & 80,4 & - & 62,1 & 64,5 & 81,4 & 62,3 & 90,3 \\
\hline & $4-11-82$ & 82,4 & 73,3 & 83,9 & - & 86,2 & 85,2 & 68,3 & 54,7 & 86,9 \\
\hline Biomasa & $27-7-82$ & 153,8 & 158,7 & 118,5 & - & 127,4 & 229,9 & 193,5 & 88,1 & 201,4 \\
\hline Fitoplancton & $21-9-82$ & 136,2 & 95,9 & 193,3 & $-m$ & 244,2 & 144,4 & 276,3 & 261,2 & 267,9 \\
\hline$m g / 1$ & $4-11-82$ & 81,8 & 88,8 & 85,7 & - & 146,9 & 121,4 & 112,0 & 88,6 & 125,8 \\
\hline
\end{tabular}

muchas similitudes con el de la estación 7. Aquí predominan los rotíferos sobre los copépodos y se da típicamente la comunidad de B. calyciflorus en Septiembre -que domina totalmente- y las especies acompañantes A. fissa y M. brachiata, muy raras en otros lugares.

Las restantes estaciones presentan una clara dominancia de B. angularis y el copépodo A.vernalis con importantes poblaciones de Polyartbra. Sin embargo no forman un grupo tan homogeneo como en el caso del fitoplancton Miracle et al. (1984) siendo claramente diferenciables por ejemplo las estaciones $4 \mathrm{~F}$ y $4 \mathrm{G}$ con afinidades con las de la zona Norte ( 5,6 y 7$)$, y algunas particularidades como una notable abundancia de $A$. girodi en Septiembre.

La evolución en el tiempo de la comunidad zooplantónica en los diversos grupos de estaciones, también es diferente. En las del Norte disminuye mucho el porcentaje de copépodos, tanto en número como en biomasa, desde Julio - en que ya estaban muy menguados- hasta Septiembre, en favor de los rotife- 
ros citados de la asociación de B. calyciflorus. En cambio en las restantes estaciones, la proporción de copépodos se mantiene más estable de Julio a Septiembre y sin embargo su biomasa aumenta ya que en este último mes disminuyen los nauplios en favor de los estadios más avanzados. Más tarde, de Septiembre a Noviembre, en las mencionadas estaciones del Norte y en las $4 \mathrm{~F}$ y $4 \mathrm{G}$, aumenta mucho la proporción de copépodos sobre todo en biomasa, loque aboga por un mayor porcentaje de adultos. Sin embargo, en las estaciones restantes disminuye su porporción aumentando la de los rotíferos, en especial, P. dolichoptera. No obstante la contribución en biomasa de los copépodos, también aumenta puesto que en este periodo predominan los adultos. Los puntos más afectados por la riada del 20 de Octubre $(1,2,3$ y 7$)$ experimentan una disminución considerable del número total de individuos.

La diversidad del zooplanton de la Albufera es muy baja, como cabía esperar en base a que está dominado por unas pocas especies. El índice de Shannon no pasa de 2 bits/individuo (Tabla 4). En el mes de Julio se dan los valores mínimos, coincidiendo con una elevada biomasa y densísimas poblaciones de B. angularis; en Septiembre y Noviembre aumenta ligeramente el valor de la diversidad al aparecer nuevas especies en la laguna y disminuir la fuerte dominancia de B. angularis y A rernalis.

Al comparar las diversas localidades, se observa una mayor diversidad en las estaciones del Sur 1, 2 y 3 que presentaban menor biomasa, mientras que los mínimos de diversidad se dan en las estaciones $4 \mathrm{~F}$ y 5 que a su vez, registraban los máximos de biomasa. Estos resultados parecen describir una relación inversa entre eutrofia y diversidad.

La Tabla 5 muestra los valores de la biomasa del zooplancton en los distintos meses y plintos de mues- treo. La biomasa media de cada mes se indica en las figs. 1 resultando una media total en el periodo estudiado de $1,8 \mathrm{mg} / 1$, uno de los valores más altos registrados en los sistemas más eutróficos del mundo (Wetzel, 1981).

La estaciones $4 \mathrm{G}, 4 \mathrm{~F}$ y 5 son las que presentan mayores valores de biomasa. En Julio ésta se debe en un $75 \%$ a los rotíferos B. angularis y B. plicatilis invirtiéndose la proporción en Noviembre en que los copépodos tienen mayor peso, coincidiendo con la preponderancia de adultos. La puntos del Sur 1, 2 y 3 tienden a presentar los menos valores de biomasa en todos los muestrarios (Tabla 5).

El zooplanton de los lagos eutróficos tiene una biomasa considerable, sin embargo la proporción relativa respecto al fitoplancton es realmente inferior a la de otros lagos. La relación de biomasas zooplanton/fitoplancton en la Albufera se situa alrededor del $1 \%$, valor muy bajo en ecosistemas acuáticos. La relación entre el número de individuos zooplancton/fitoplancton es aun mucho más baja, del orden de 2 por millón (Tabla 5). El fuerte desplazamiento de ambas relaciones en favor del fitoplancton puede interpretarse en base a que éste está constituido por cianobacterias hasta en un $90 \%$, ya que estas algas resultan un alimento poco adecuado cuando no tóxico para el zooplancton.

\section{CONCLUSIONES}

La densidad y biomasa del zooplacton alcanzan en la Albufera valores muy elevados, de los más altos entre los obtenidos en otros sistemas hipertróficos, llegando a más de 5.000 organismos/litro y casi $\mathrm{S}$ miligramos/litro respectivamente. La relación de biomasas zooplancton/fitoplancton es del $1 \%$

Tabla IV - Indices de diversidad zooplanctónica (bits/indisiduos para cadal localidad y dia y medias respectivas

Zoxplankend diversits indexes for each statem and date and theil respectere means

\section{Estación Media}

\begin{tabular}{|c|c|c|c|c|c|c|c|c|c|c|}
\hline Fecha & 1 & 2 & 3 & $4 G$ & $4 \mathrm{~F}$ & 5 & 6 & 7 & 8 & mensual \\
\hline $27-7-82$ & 1,75 & 1,56 & 1,65 & - & 1,21 & 1,30 & 1,63 & 1,53 & 1,53 & 1,52 \\
\hline $21-9-82$ & 1,86 & 1,80 & 1,83 & $\mathrm{~T}, 88$ & 1,85 & 1,31 & - & 1,95 & 1,75 & 1,77 \\
\hline $4-11-82$ & 1,82 & 2,04 & 2,00 & 1,71 & 1,68 & 1,67 & 1,82 & 1,34 & 1,73 & 1,75 \\
\hline $\begin{array}{l}\text { Media por } \\
\text { estación }\end{array}$ & 1,81 & 1,80 & 1,82 & 1,79 & 1,58 & 1,42 & 1,72 & 1,60 & 1,67 & - \\
\hline
\end{tabular}


作 Tabla V.-Biomasa total del zooplancton $(\mathrm{mg} /$ ) y contribucón fitoplancton/zooplancton en biomasa y número de individuos. durante el periodo de estudio. Se indica tambien la relacion fioplane different stations and sampling Total zooplankton biomass $(\mathrm{mg} / \mathrm{l}$ ) and relative contribution of the main zooplanktonic groups in the diff dates. The ratios phytoplankton/zooplankton in biomass and number of individuals are also indicared.

\begin{tabular}{|c|c|c|c|c|c|c|c|c|}
\hline \multirow[b]{3}{*}{ Estación } & \multirow[b]{3}{*}{ Fecina } & \multirow{3}{*}{$\begin{array}{l}\text { Sionasa } \\
(m g / 1)\end{array}$} & \multirow{3}{*}{$\begin{array}{l}\text { Media por } \\
\text { estación }\end{array}$} & \multirow{2}{*}{\multicolumn{3}{|c|}{$\begin{array}{l}\text { Contribución de cada grupo } \\
\text { a la biomasa (en } t_{0} \text { ) }\end{array}$}} & \multicolumn{2}{|c|}{ Rels. Fitoplancton/zooplancton } \\
\hline & & & & & & & Relación & Relación \\
\hline & & & & tucladóceros & zCopépodos & thotiferos & & \\
\hline \multirow{3}{*}{1} & $27-7-32$ & 1.69 & \multirow{3}{*}{1,45} & 0 & 56,4 & 43,6 & 1,10 & 0,73 \\
\hline & $21-9-82$ & 2,36 & & 5,8 & 79,8 & 14,4 & 1,73 & 2,31 \\
\hline & $4-11-32$ & 0.31 & & 0 & 81.8 & 18,2 & 0,37 & 0.80 \\
\hline \multirow{3}{*}{2} & $27-7-32$ & 2.03 & \multirow{3}{*}{1,06} & 0 & 48,8 & 51,2 & 1,28 & 1,89 \\
\hline & $21-9-82$ & 0,38 & & 5.1 & 67,2 & 27,7 & 0,91 & 1,32 \\
\hline & $411-82$ & 0.29 & & 0 & 62,7 & 37,3 & 0,33 & 1,29 \\
\hline \multirow{3}{*}{3} & $27-7-82$ & 2,13 & \multirow{3}{*}{1,37} & 0 & 54,3 & 45,7 & 1,80 & 1,80 \\
\hline & $21-3-82$ & 1.56 & & $11, ?$ & 58,4 & 29,9 & 0,81 & 1,50 \\
\hline & $411-32$ & 0,42 & & 0 & 78,7 & 21,3 & 0,50 & 1.14 \\
\hline \multirow{2}{*}{$4 G$} & $21-9-92$ & 2,94 & \multirow{2}{*}{1,96} & 4,7 & 26,7 & 68,6 & & \\
\hline & $4-11-82$ & 0,98 & & 0 & 86,8 & 13,2 & & \\
\hline \multirow{3}{*}{$4 F$} & $2 i-7-82$ & 4,97 & \multirow{3}{*}{2,90} & 0 & 24,3 & 75,7 & 3,90 & 5,42 \\
\hline & $21-9-82$ & 2,19 & & 12.5 & 34,4 & 53,1 & 0,90 & 2,23 \\
\hline & $411-32$ & 1,34 & & 0 & 36,8 & 13,2 & 1,05 & 1,66 \\
\hline \multirow{3}{*}{5} & $27-7-82$ & 4,32 & \multirow{3}{*}{2,97} & 0 & $23, i$ & 76.9 & 1,88 & 3,08 \\
\hline & $21-9-82$ & 2.75 & & 29,8 & 2.6 & 67,6 & 1,91 & 5,54 \\
\hline & $4-11-82$ & 1,80 & & 0 & 72,1 & 27,9 & 1,49 & 3,30 \\
\hline \multirow[t]{2}{*}{6} & $27-7-82$ & 2,13 & \multirow{2}{*}{1,65} & 0 & 24,0 & 76,0 & 1,10 & 1,28 \\
\hline & $4-11-82$ & 1,17 & & 0 & 85,9 & 14,1 & 1,05 & 1,73 \\
\hline \multirow{3}{*}{7} & $27-7-82$ & 0,99 & \multirow{3}{*}{1,20} & 0 & 27,6 & 78,4 & $T .12$ & 1,15 \\
\hline & $21-9-82$ & 2,30 & & 55,7 & 18,9 & 25,3 & 0,88 & 0,99 \\
\hline & $411-82$ & 0,30 & & 0 & 78,1 & 21,9 & 0,34 & 0,99 \\
\hline \multirow{3}{*}{8} & $27-7-32$ & 3,58 & \multirow{3}{*}{1,90} & 0 & 38,1 & 61,9 & 1,78 & 2,54 \\
\hline & $21-9-82$ & 1,23 & & 4,6 & 55,4 & 40.0 & 0,47 & 0,81 \\
\hline & $4-11-82$ & 0,89 & & 0 & 75,9 & 24,1 & 0,71 & 1,80 \\
\hline
\end{tabular}

cuando los valores que más frecuentemente presentan los lagos se situan entre el 10 y $15 \%$, lo que se atribuye a la elevada proporción de cianobacterias.

Existe una clara variación temporal zooplanctónica evidenciada por la correlación estadística, que delimita tres grupos de especies: permanentes, de finales de verano y de otoño. La sucesión de estos grupos parece obedecer, en primer lugar, a la renovación del agua de la Albufera en septiembre, consecuencia de la apertura de las compuertas y drenaje de los arrozales y, en segundo lugar, al importante flujo de agua que penetró en la laguna a causa de las catastróficas inundaciones ocurridas durante el periodo de estudio.

La variabilidad del zooplancton en las diversas partes de la laguna, es menos ostensible que la variación temporal. Sin embargo en las estaciones de la zona Norte -puntos 5, 6 y 7 que presentaron mayor nivel de eutrofia y mineralización del agua y una menor proporción de cianobacterias en favor de las diatomeas- se diferencian por una mayor dominancia de los Branchionus: B. plicatilis y B. calyciflorus en vez del característico $B$. angularis dominante en el resto de la laguna. Asi mismo se observa en dichas estaciones una menor proporción del copepodo en favor de los cladóceros - muy raros en otras partes de la AlbuferaM. brachiata, en este periodo de estudio y Daphia magna en primavera, según datos de estudios inéditos posteriores de los mismos autores.

La diversidad de la comunidad zooplanctónica es baja, aumentando tras una perturbación -incremento 
del flujo de agua en septiembre y noviembre- al contrario de lo que sucede en los ecosistemas no sometidos a un estado de máximo stress.

\section{AGRADECIMIENTOS}

Este trabajo ha sido realizado gracias a las facilidades prestadas por el Excm. Ayuntamiento de Valenica y a la subvención otorgada por la CAICYT (proyecto) $\mathrm{n}^{\mathrm{Q}} 915 / 81$ )

\section{BIBLIOGRAFIA}

Arévalo, C. 1916. Cladóceros del plancton de la Albufera de Valencia Anales del Instituto General y Técnico de Valencia Trabaio del Laboratorio de Hidrobiología Vol !. núm 1

A révalo, C. 1918. Algunos rotíferos planktónicos de la Albufera de Valencia Anales del Inscituto General y Técnico de Valencia Trabajo del Laboratorio de Hidrobiología. Vol II, núm 4

Blanco, C. 1974́. Estudio de la contaminación de la Albufera de Valencia y de los efectos de dicha contaminación en la fauna y en la flora del lago. Tesis Doctoral Universidad de Valencia

Edmondson. W. T. \& G. G. Winberg, 1971. A Manual on methods for the Assessmenr of Secondary Productivity in Fresh
Waters ISP Handbook núm 17. Blackwell Oxford and Edinburgh Golterman, H.I.. R.S. Clymo \& M. Ohnstad. 1978. Methods for Physical and Chemical Analysis of Fresh Warers IBP Handbork num 8 Blackwell Oxford, Edinburgh, london, Melbourne Koste, W. 1978. Rutatoria Die Radertiere Mitteleuropasbegrudet von Max Voigr Uberordnung Monogononta Gebrüder Bortaeger Berlín

Miracle, M.R. 1974. Niche estructure in fres water zooplankton: a principal components aproach Ecology 55: 1306-1316.

Miracle M.R., García, P. y Vicente, E. 1984. Heterogeneidad espacial de las comunidades fitoplanctónicas de la Albufera de Valencia. Limnética 1:20 - 31

Pardo, L. 1942. La Albufera de Valencia Estudio I.imnológico, Biológico, Económico y Antropológico Biología de las aguas continentales Il Instituto Forestal de Investigaciones y Experien cas núm 2. Madrid

Ruttner-Kolisko, A. 1974. Plankton Rotifers Biology and Taxonomy Die Binnegewilsser Vol XXVI purt 1

Ruttner-Kolisko, A. 1977. Sggestions for biomass calculation of plankton rotifers Arch Hydrobiol Beih. Ergebii Limnol.8 71.75 Serra, M.; Miracle, M.R. y Vicente E. 1984. Interrelaciones entre los principales parámetros limnológicos de la A lbuferal de Valencia Limnética 1:9 - 19

Wetzel. R.G. \& G.E. Likens. 1979. Limnological Analyses W B Saunders company Philadephia, London. Toronto Wetzel, R.G. 1981. I.imnología Omega Barcelonal 